\title{
Millimetron-a large Russian-European submillimeter space observatory
}

\author{
Wolfgang Wild • Nikolay S. Kardashev •
}

On behalf of the Millimetron consortium:

S. F. Likhachev • N. G. Babakin • V. Y. Arkhipov •

I. S. Vinogradov • V. V. Andreyanov •

S. D. Fedorchuk • N. V. Myshonkova •

Y. A. Alexsandrov • I. D. Novokov •

G. N. Goltsman - A. M. Cherepaschuk •

B. M. Shustov - A. N. Vystavkin - V. P. Koshelets •

V. F. Vdovin • Th. de Graauw • F. Helmich •

F. vd Tak • R. Shipman • A. Baryshev • J. R. Gao •

P. Khosropanah • P. Roelfsema • P. Barthel •

M. Spaans • M. Mendez • T. Klapwijk • F. Israel •

M. Hogerheijde • P. vd Werf • J. Cernicharo •

J. Martin-Pintado • P. Planesas • J. D. Gallego •

G. Beaudin • J. M. Krieg • M. Gerin • L. Pagani •

P. Saraceno - A. M. Di Giorgio • R. Cerulli •

R. Orfei • L. Spinoglio • L. Piazzo • R. Liseau •

V. Belitsky • S. Cherednichenko • A. Poglitsch •

W. Raab • R. Guesten • B. Klein • J. Stutzki •

N. Honingh $\cdot$ A. Benz $\cdot$ A. Murphy $\cdot$ N. Trappe $•$

A. Räisänen

Received: 31 October 2007 / Accepted: 10 April 2008/Published online: 11 July 2008

(C) Springer Science + Business Media B.V. 2008

\begin{abstract}
Millimetron is a Russian-led $12 \mathrm{~m}$ diameter submillimeter and far-infrared space observatory which is included in the Space Plan of the Russian Federation for launch around 2017. With its large collecting area and state-of-the-art receivers, it will enable unique science and allow at least one order of magnitude improvement with respect to the Herschel Space Observatory. Millimetron will be operated in two basic observing modes: as a single-dish observatory, and as an element of a ground-
\end{abstract}

\footnotetext{
W. Wild $(\bowtie)$

Low Energy Astrophysics, SRON Netherlands Institute for Space Research, Landleven 12, 9747 AD Groningen, The Netherlands e-mail: W.Wild@sron.rug.nl
}

Th. de Graauw • F. Helmich • F. vd Tak • R. Shipman • A. Baryshev • P. Khosropanah • P. Roelfsema SRON Netherlands Institute for Space Research, Landleven 12, 9747 AD Groningen, The Netherlands 
space very long baseline interferometry (VLBI) system. As single-dish, angular resolutions on the order of 3 to 12 arc sec will be achieved and spectral resolutions of up to a million employing heterodyne techniques. As VLBI antenna, the chosen elliptical orbit will provide extremely large VLBI baselines (beyond 300,000 km) resulting in micro-arc second angular resolution.

Keywords Submillimeter - Space observatory · Radio lines: general · Space instrumentation · Techniques: spectroscopic telescopes · VLBI

\section{Introduction}

Millimetron is a large (12 $\mathrm{m}$ diameter) space observatory for millimeter, submillimeter (submm) and far-infrared (far-IR) observations. This Russian-led mission will enable astronomers to observe the universe with unprecedented sensitivity and angular resolution. The far-infrared and submillimeter spectral bands are crucial regimes for the study of the formation and evolution of stars, planets, and galaxies. In addition, extremely high-angular resolution imaging by very long baseline interferometry (VLBI) allows exploration of ultra-compact radio sources including black holes.

W. Wild $\cdot$ P. Barthel $\cdot$ M. Spaans $\cdot$ M. Mendez Kapteyn Astronomical Institute, University of Groningen, Landleven 12, 9747 AD Groningen, The Netherlands

N. S. Kardashev $\cdot$ S. F. Likhachev $\cdot$ N. G. Babakin $\cdot$ V. Y. Arkhipov I. S. Vinogradov V. V. Andreyanov $\cdot$ S. D. Fedorchuk $\cdot$ N. V. Myshonkova $\cdot$ Y. A. Alexsandrov $\cdot$ I. D. Novokov Astro Space Centre of P.N. Lebedev Physical Institute, Moscow, Russia

G. N. Goltsman

Moscow State Pedagogical University, Moscow, Russia

\section{A. M. Cherepaschuk}

Sternberg Astronomical Institute, Moscow State University, Moscow, Russia

B. M. Shustov

Institute of Astronomy of Russian Academy of Sciences, Moscow, Russia

A. N. Vystavkin • V. P. Koshelets

Institute of Radio Engineering and Electronics, Moscow, Russia

V. F. Vdovin

Institute of Applied Physics, Nizhny Novgorod, Russia

J. R. Gao

SRON Netherlands Institute for Space Research, Delft Technical University, Sorbonnelaan L, 3584 CA Utrecht, The Netherlands

T. Klapwijk

Delft Technical University, Delft, The Netherlands

F. Israel $\cdot$ M. Hogerheijde $\cdot$ P. vd Werf

University of Leiden, Leiden, The Netherlands

Springer 
Millimetron has two scientific observing modes each of which is unique and represents a major step forward in the investigation of the universe we are living in. Firstly, Millimetron will be used as a $12 \mathrm{~m}$ diameter single-dish space observatory for high-sensitivity and high angular resolution observations of the submillimeter universe. Secondly, Millimetron will be used as a VLBI antenna in millimeter and submillimeter wavelength bands providing extreme angular resolution of better than one micro-arc second. Observations in both observing modes will substantially contribute to solving questions as outlined in the Cosmic Vision themes.

Millimetron (Spectrum-M) is part of the Space Plan of the Russian Federation with a planned launch in 2017. The Russian Space Agency has approved the mission and has allocated funds for the development and implementation of Millimetron (Spectrum-M).

J. Cernicharo $\cdot$ J. Martin-Pintado

Departamento De Astrofísica Molecular E Infrarroja, CSIC, Madrid, Spain

P. Planesas · J. D. Gallego

Centro Astronomico de Yebes, Observatorio Astronomico Nacional, Guadalajara, Spain

G. Beaudin · J. M. Krieg

LERMA, Observatoire de Paris, Paris, France

M. Gerin · L. Pagani

LESIA, Observatoire de Paris, Meudon, France

P. Saraceno - A. M. Di Giorgio · R. Cerulli • R. Orfei • L. Spinoglio

Istituto di Fisica dello Spazio Interplanetario (INAF), Rome, Italy

L. Piazzo

University La Sapienza, Rome, Italy

R. Liseau

Onsala Space Observatory, Onsala, Sweden

V. Belitsky $\cdot \mathrm{S}$. Cherednichenko

Chalmers University of Technology, Göteborg, Sweden

A. Poglitsch • W. Raab

Max-Planck-Institut für Extraterrestrische Physik, Garching, Germany

R. Guesten • B. Klein

Max-Planck-Institut für Radioastronomie, Bonn, Germany

J. Stutzki $\cdot$ N. Honingh

KOSMA, University of Cologne, Cologne, Germany

A. Benz

Institut für Astronomie, Eidgenössische Technische Hochschule Zürich, Zürich, Switzerland

A. Murphy $\cdot$ N. Trappe

National University of Ireland Maynooth, Maynooth, Ireland

A. Räisänen

Radio Laboratory, TKK Helsinki University of Technology, Helsinki, Finland 
At present, the Astro Space Center (ASC) in Moscow is carrying out studies for Millimetron which are funded by the Russian Space Agency. Concerning Millimetron, ASC has established contacts with the European Space Agency (ESA), European Southern Observatory (ESO), and lately with SRON Netherlands Institute for Space Research with the aim to explore possibilities for European participation in the mission.

\section{Science with Millimetron}

The $12 \mathrm{~m}$ dish of Millimetron can be used in two observing modes: (1) Single-dish submillimeter/far-infrared observations, and (2) Space-ground VLBI (SVLBI) observations at (sub-) millimeter wavelengths. We will give a brief overview of the main science goals for each of these observing modes.

\subsection{Introduction: the formation of stars and galaxies}

Two prime foci of 21 st century astrophysics are the studies of the obscured universe and the molecular universe, at far-infrared and (sub-)millimeter wavelengths. The reason is that only through such studies, which target cool dust and gas, we can obtain crucial information about the formation processes of stars and galaxies, the central issues in astrophysics. Millimetron will permit to make the important next steps in spatial resolution as well as sensitivity.

Following up on the Infrared Astronomical Satellite (IRAS) mission, the Infrared Space Observatory (ISO) and Spitzer have subsequently truly opened the dusty and molecular universe. These missions observed in great detail the processes involved in small- and large-scale star-formation, at mid- and far-infrared wavelengths. ISO revealed the presence of cold $(15 \mathrm{~K})$ dust in galaxies and established the unexpected fast decay of starburst episodes. Mid- and far-infrared luminosities, colors, and spectra of the [CII] and [OI] cooling provided insight in the physics of star formation. Imaging of the warm dust in nearby galaxies proved to be a fine tool: ISO revealed fascinating cases of obscured star-formation such as in the Antennae galaxies NGC4038/39 (Fig. 1). The spectroscopic capabilities of ISO and Spitzer permitted great advance in our understanding of the gas properties of nearby dusty and gas-rich objects, including the important class of ultraluminous infrared galaxies (ULIRGs). The magnificent spectra obtained with the Spitzer infrared spectrograph provide conclusive evidence for the starburst-active galactic nuclei symbiosis (as suggested from ISO observations) in a variety of extragalactic objects.

In space, a far-infrared interferometer (FIRI, Helmich and Ivison, this issue [3]) is still far beyond the horizon, and larger antennas must make the necessary next step. If such a telescope delivers improved detector sensitivity at the same time, its advantages are even greater. The Millimetron mission will be able to deliver all this. Its sensitive receivers and its large collecting area will allow at least one order of magnitude improvement with respect to Herschel. Whereas Herschel will study the formation of stars and galaxies in a global fashion, Millimetron will zoom in on the processes, and observe fainter, more distant objects. The development of Millimetron would therefore be a wonderful far-infrared parallel to the submillimeter developments on the Chajnantor plateau! 
Fig. 1 Antennae galaxies NCG4038/39 (Spitzer Space Telescope)

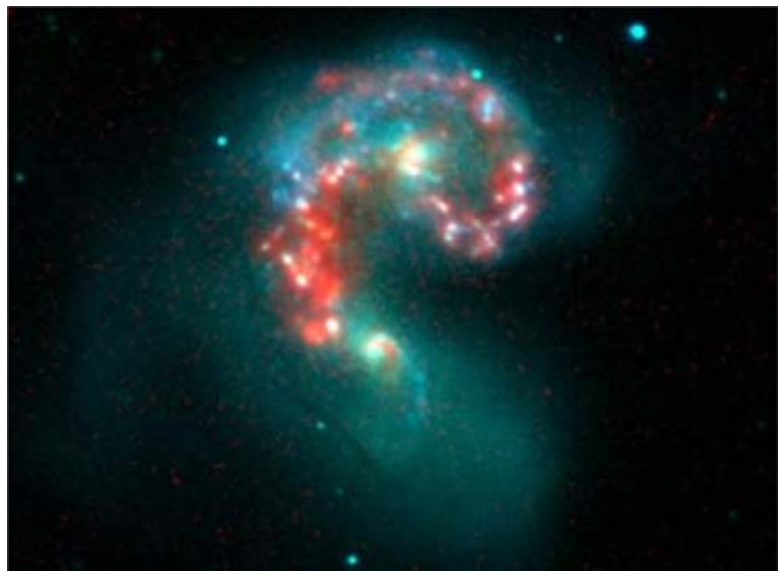

\subsection{Diagnostic features in the far-infrared regime}

The far-IR and submm regions are crucial regimes because they reveal the radiative response of gas and dust in galaxies to the input of energy from stars (photon absorption) or by the dissipation of mechanical energy (shocks). The resulting spectrum contains the fingerprints of the astrophysical processes governing the formation of stellar populations and of supermassive black holes. Unfortunately, the Earth's atmosphere is opaque in essential regions of the far-IR/submm regime, such as the region from 50 to $200 \mu \mathrm{m}$ which contains the fundamental interstellar gas cooling lines [CII] $158 \mu \mathrm{m}(1,900 \mathrm{GHz})$ and [OI] $63 \mu \mathrm{m}(4,700 \mathrm{GHz})$. By providing observing capability in this region at unprecedented resolution (3-4") and sensitivity, Millimetron will revolutionize this field (Table 1).

\subsection{Spatially resolving the cosmic IR background}

The Cosmic Infrared Background (CIRB) is a fundamental component of the present radiation content of the Universe and is made essentially of the long wavelength

Table 1 Main spectral features targeted by Millimetron

\begin{tabular}{|c|c|c|c|}
\hline Line & Frequency $(\mathrm{GHz})$ & Goal & Competitive edge \\
\hline $\begin{array}{l}\mathrm{H} 2 \mathrm{O} 1(10)-1(01) \& \\
1(11)-0(00)\end{array}$ & $556.9 \& 1,113.3$ & Tracers of star formation & $\begin{array}{l}\text { Superior sensitivity and spatial } \\
\text { resolution wrt Herschel }\end{array}$ \\
\hline Dust continuum & $500-5,000$ & $\begin{array}{l}\text { Mass and temperature } \\
\text { diagnostic }\end{array}$ & $\begin{array}{l}\text { Superior sensitivity and spatial } \\
\text { resolution wrt Herschel }\end{array}$ \\
\hline $\mathrm{p}-\mathrm{H} 2 \mathrm{D}+1(01)-0(00)$ & $1,370.1$ & $\begin{array}{l}\text { Unique tracer of protostellar } \\
\text { condensations }\end{array}$ & $\begin{array}{l}\text { Superior sensitivity and spatial } \\
\text { resolution wrt Sofia }\end{array}$ \\
\hline [CII] 2P(3/2)-2P(1/2) & $1,900.5$ & $\begin{array}{l}\text { Main coolant of interstellar } \\
\text { gas }\end{array}$ & $\begin{array}{l}\text { Superior sensitivity and spatial } \\
\text { resolution wrt Herschel }\end{array}$ \\
\hline $\mathrm{HD} \mathrm{J}=1-0$ & $2,675.0$ & $\begin{array}{l}\text { Origin of molecular clouds } \\
\text { and giant planets }\end{array}$ & $\begin{array}{l}\text { Superior sensitivity and spatial } \\
\text { resolution wrt Sofia }\end{array}$ \\
\hline [OI] 3P(1)-3P(2) & $4,744.8$ & $\begin{array}{l}\text { Tracer of heating by shocks } \\
\text { and UV }\end{array}$ & $\begin{array}{l}\text { Superior sensitivity and spatial } \\
\text { resolution wrt Sofia }\end{array}$ \\
\hline
\end{tabular}


output from all sources throughout the history of the Universe from the present time till the epoch of recombination, only 400,000 years after the Big Bang. In contrast, the Cosmic Microwave Background (CMB), dominating the radiation spectrum for frequencies lower than $800 \mathrm{GHz}$, has been produced in the hot and dense phases of the universe before the recombination. Note that the very different spectra of the CIRB with respect to the CMB allow them to be relatively easily separated for wavelengths shorter than $1 \mathrm{~mm}$, and in this wavelength regime the CIRB dominates the galactic emission by a factor four in the lowest cirrus regions.

Our current understanding of the CIRB considers it to be the integral of the reprocessed light by dust during phases of enhanced activity in galaxies and active nuclei. Dust, which is observed to be ubiquitous in galactic star-forming regions and star-forming galaxies in general, is likely to have been present also during the major past events of the formation of stellar populations. According to this interpretation, the long-sought primeval galaxies would be undetectable in the optical, and would have left an important trace in the CIRB. This indicates that the most important phases of the formation of galaxies and their nuclear supermassive black holes (responsible for the nuclear activity) might only be investigated in the far-infrared and in the sub-mm, where the CIRB peaks.

A crucial theme of observational cosmology for the next several years will then be this exploration of the far-IR \& sub-mm sky in the attempt to identify and characterize the enigmatic sources of the CIRB. Figure 2 is a model representation illustrating the capabilities of future long-wavelength space missions (nothing can be done unfortunately from ground in this spectral domain) to resolve this background, in a similar fashion as it was done for example for other extragalactic relics like the $\mathrm{X}$-ray or radio backgrounds.

Limited sensitivity and resolution make it impossible to resolve the CIRB with the forthcoming Herschel mission or future space observatories like the Space Infrared Telescope for Cosmology and Astrophysics (SPICA): all these will produce confusion-limited images able to resolve the CIRB only at its short wavelength boundary. Similar considerations apply to its long wavelength $(\lambda>500 \mu \mathrm{m})$ boundary accessible by ground-based millimetric observatories.

The spectral capabilities of Millimetron will allow a spectral characterization of these sources through redshifted fine-structure emission lines characteristic of either

Fig. 2 The CIRB spectrum and fractions of it that will potentially be resolved by future missions (HSO, SPICA). Only a large (10-12 m) diffractionlimited space observatory, like Millimetron, would completely resolve the CIRB into sources

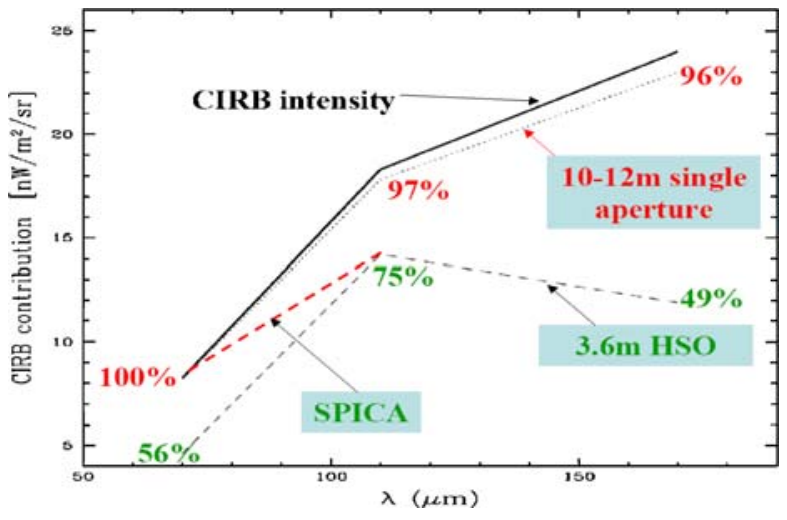


stellar photoionization ([NeII], [SIII] lines) or photoionization by the hard continuum of an active galactic nucleus ([OIV], [NeV]). It will thus be able to trace directly the coeval buildup of stellar population and black hole with redshift in galaxies of a range of luminosities. Millimetron will thus provide the currently missing window on the region of photoionized gas, and provide direct complementarity to ALMA, which will probe principally the neutral molecular gas in the same objects.

\subsection{Star-forming galaxies at low redshift}

Low-redshift galaxies form crucial laboratories for studying the physical processes occurring at higher redshifts in detail. By probing the neutral star-forming medium, we probe both the conditions in the prestellar gas phase and the feedback of the star formation (or black hole accretion) process on the ambient medium. Here Millimetron provides unique insight: the $\mathrm{H}_{2} \mathrm{O}$ ground-state transition forms a probe of warm, dense, cooling molecular gas that is not available from the ground. Simultaneously, the $[\mathrm{CII}]$ and $[\mathrm{OI}]$ lines probe the more extended lower density medium. The change in cooling balance from quiescent to actively star-forming galaxies such as ULIRGs is shown by the decreasing importance of [CII] cooling with increasing luminosity. For instance, in ULIRGs the cooling by the CO rotational lines approximately equals that in [CII], indicating a totally different thermal balance than in lower luminosity objects [8]. While Herschel will be able to skim the surface of this subject, what is really required is a study of fainter galaxies, both locally and at higher redshift, in order to establish the cooling properties as a function of physical parameters such as galaxy luminosity, type, environment, metallicity, presence (or not) of an active nucleus, and others.

\subsection{Far-infrared spectroscopy of star-forming galaxies}

With a resolution of 3-4 arc sec at the wavelengths of the important [OI] and [CII] cooling lines, the medium-resolution (imaging) spectrograph on Millimetron will be able to trace accurately the location of the main cooling radiation and therefore characterize the thermal balance of the interstellar material of galaxies at a resolution comparable to seeing-limited observations in the visual regime. At the distance of the nearest ULIRG (Arp220, D=74 Mpc) the Millimetron resolution at $60 \mu \mathrm{m}$ corresponds to $\sim 1,000 \mathrm{pc}$, sufficient to resolve the central concentration and gas and dust where most of the luminosity originates. Given the linewidths of galactic nuclei, a spectral resolution $R=3,000$ is sufficient for revealing the kinematic structure of the emitting gas.

Finally, an attractive application of Millimetron is the search for primeval (metalfree) galaxies. In the absence of metals a cloud of molecular gas must cool through $\mathrm{H}_{2}$ line emission and the rotational $\mathrm{H}_{2}$ lines will be redshifted into the Millimetron regime. This process has been modeled in detail by Mizusawa et al. [6] who demonstrate that for a cloud contracting at a rate (expressed as a star formation rate) of $100 \mathrm{M}_{\text {sun }} /$ year a flux of $10^{-23} \mathrm{~W} / \mathrm{m}^{2}$ is expected in the brightest $\mathrm{H}_{2}$ line for a source at $z=6$ (presumable the end of reionization). If such sensitivities can be reached, Millimetron could for the first time detect population III (metal-free) galaxies, in the era just before or during cosmic reionization. While a blind search 
for such galaxies could be prohibitively time-consuming, targets may be preselected from Ly-alpha narrow-band imaging (since such galaxies are expected to be significant Ly-alpha emitters) or from high-z Gamma-Ray-Burst surveys.

\subsection{Physical conditions in Galactic regions of star formation}

The heating of interstellar gas reflects the action of powerful shocks driven by jets and outflows from young stellar objects. Alternatively, UV photons from nearby young massive stars photodissociate and heat the surrounding gas, forming a photon dominated region. Both of these types of environments are cooled by infrared atomic fine structure line emission.

Our understanding of the physics of interstellar clouds is clearly incomplete as shown by our inability to model the excitation conditions in the cold [C II] and warm [O I] media. Local emission regions and intrinsic line widths, whether galactic or extragalactic, are expected to be small. Progress on the observational side therefore clearly requires significantly increased spectral and spatial resolution compared to what will be offered in the foreseeable future by, e.g., Herschel and/or the Stratospheric Observatory for Infrared Astronomy (SOFIA). A large telescope as that proposed for Millimetron and equipped with heterodyne receivers would be able to fulfill these requirements. For external ultra-luminous galaxies, the sensitivity provided by a large collecting area in combination with an intermediate-resolution spectrometer for far infrared wavelengths would be well adapted to study the [O I] $63 \mu \mathrm{m}$ emission for large redshifts, $z>3$, and hence match the upcoming (lower spatial resolution) observations of the [C II] $157 \mu \mathrm{m}$ line with Herschel.

\subsection{Formation of molecular clouds and stars}

A large single dish far-infrared telescope equipped with array receivers such as Millimetron is also the ideal tool for studying the formation of molecular clouds and stars. Stars are born in dense cold cores inside molecular clouds, but the life time of these cores and their formation within molecular clouds, as well as the formation of molecular clouds themselves, are far from understood. Numerical simulations by Hennebelle et al. [4] and Glover and Mac Low [2] have shown that molecular clouds can be formed out of diffuse atomic interstellar gas, and that the structure of the clouds is both a consequence of the early structure of the atomic gas as well as the condensation process. Furthermore, while the formation of molecular hydrogen itself can be accelerated by the shocks driven by turbulence, the formation of $\mathrm{CO}$ and other stable molecules requires longer times, as these molecules are easily photodissociated. Therefore, significant abundances are only achieved in regions shielded from UV radiation. HilyBlant and Falgarone [5] propose that dense molecular cores are indeed related to more diffuse atomic and molecular gas, from which the cores accrete material and gain mass.

As the main coolants of diffuse matter, the $[\mathrm{CII}]$ and [OI] lines are the best probes to study the process of core formation in the interstellar medium and test the above theories. Disentangling the respective roles of turbulence, gravity and magnetic fields requires large scale maps at high spectral and spatial resolution, as small scale structures are known to exist down to arc second scale in the diffuse interstellar medium. The role of the magnetic field will be revealed by the shape of the 
structures, but it is also expected that the line profiles will bear an imprint of the magnetic field, because the velocity field of the molecular gas will be affected.

Prior to star formation, the molecular gas becomes very cold in the interior of dense molecular cores, and most of the heavy molecular species get frozen on grains. Since the substitution of a hydrogen atom by a deuterium atom is favored at low temperatures, deuterated molecules become very abundant, and are the best tools to study the physical conditions, gas dynamics and chemistry in these environments. The recently detected molecular ions $\mathrm{H}_{2} \mathrm{D}+$ and $\mathrm{D}_{2} \mathrm{H}+$ are key actors in the deuterium fractionation process. While a single transition of either ion is accessible from the ground with great difficulty, Millimetron will routinely map both ions, offering unique diagnostic capabilities of the initial conditions and first steps of star formation. Measurements of the HD rotational lines at 112 and $56 \mu \mathrm{m}$ would provide an ideal complement, as HD is the main deuterium reservoir. While the first detection of the rotational lines have been obtained by ISO, the diagnostic capabilities of HD have been demonstrated by Spitzer. Neufeld et al. [7] have mapped the HD emission from supernova shocks, and show that the $R(3) / R(4)$ ratio is a probe of gas pressure. HD lines probe the deuterium reservoir, and trace the presence of molecular gas accurately.

The onset of actual star formation in the molecular condensations is best traced by the water molecule. Emission in its ground state lines at 557 and $1113 \mathrm{GHz}$ is a clear sign of dense gas that has been heated up by nearby young stars. While Herschel will excel in the study of water in advanced stages of star formation, the weakness of the emission and small size of the emitting region in the very first phases of stellar activity call for the sensitivity and resolution of Millimetron.

\subsection{Protoplanetary disks}

The past decade has witnessed the discovery of a multitude of planetary systems, exhibiting a wide variety of properties. This shows that our Solar System is neither unique nor typical. Understanding how this richness arises from the formation process of planets will occupy astronomers for the next decades. It will require observations of sufficient spatial resolution that can penetrate the dense layers of dust present in circumstellar disks, the birth sites of planets.

Water The water molecule is a key player in protoplanetary disks. Expected to be frozen out onto dust grains in cold regions $(<100 \mathrm{~K})$, it should be present in the gas phase in the disk atmosphere and the inner few AU of the disk. In these latter regions it is a key player in the cooling balance of the gas, and its presence in the terrestrial planet formation zone is crucial for the formation of life. While Herschel will bring the first opportunity to detect the ground-state water lines from protoplanetary disks, the limited sensitivity and resolution will at best permit detections in a few exceptional cases. The much larger Millimetron dish will enable systematic studies of the water content of protoplanetary disks in nearby star-forming regions, and thus put the study of habitable planet formation on a solid footing.

Debris disks After planet formation, the remnant dust particles settle in so-called debris disks. The classical example is the tenuous disk around Vega, discovered by IRAS. The properties of such disks are important constraints on theories of planet 
formation, but are not well known from observations. The superior sensitivity and resolution of Millimetron relative to Herschel allow us to detect the dust and [CII] emission from debris disks out to large distances, and permit to study their physical properties in a statistically significant sample.

\subsection{Solar system science}

A large submillimeter antenna in space like Millimetron would be an important tool to study the water meteorology in planetary atmospheres and the water activity in comets. The small antenna beam at submillimeter wavelengths allows mapping the water lines on planets and comets, and by repeating these observations regularly, we may establish the meteorological system and probably even start with climate measurement. The atmospheres of planets and moons in our Solar System, as well as comets are the prime targets for spectroscopic observations.

A key contribution of Millimetron will be the measurement of the HD ground state line in the atmospheres of the giant planets. This line probes the deuterium abundance, a very important measurement for understanding the formation of planets and the origin of the solar system. The HD abundance in the atmospheres of Saturn and Jupiter constrains the place in the Galaxy where our Sun has formed. While SOFIA and Herschel will assess this topic for the first time, Millimetron will map the HD abundance over the planetary disks, and directly test the origin assumption. One expects that on Uranus and Neptune, the $\mathrm{D} / \mathrm{H}$ ratio is enhanced by mixing of their hydrogen envelopes with D-enriched grains and this process could also be at play on Saturn and Jupiter. Maps of the four giant planets in HD will thus permit the determination of the composition and origin of the grains in the primitive Solar nebula.

\subsection{Millimetron as VLBI antenna: Space-ground VLBI science}

In addition to single-dish observations, Millimetron will also operate as a space-ground Very Large Baseline Interferometer providing unprecedented angular resolution.

The main scientific goal of the Millimetron mission in Space VLBI mode operation will be the exploration, with extreme high angular resolution (better than 1 $\mu \mathrm{m}$ arc sec) of extremely compact radio sources. The list of the expected results includes:

(1) first direct imaging of the event horizon of super-massive black holes in galaxies at different redshifts, particularly for the black hole at the center of our Galaxy; for these super-massive black holes and for stellar-mass black holes (microquasars) in our Galaxy and other galaxies in the local group, Millimetron will further study the physical parameters and processes near the black-hole horizon;

(2) determine the cosmological evolution (by measuring black holes at different redshift) of black holes parameters at the center of galaxies;

(3) first direct study of particle acceleration regions very close to a black hole;

(4) first measurements of proper motion and cosmological proper motion and parallaxes of such objects;

(5) first measurements of the dynamics of close binary quasars; 
(6) first direct imaging of structures, physical parameters and process near the surface of neutron stars by observation of pulsars and magnetars with inverted radio spectrum;

(7) the first detection of structure, and study of the evolution of this structure, of gamma-ray bursts radio afterglows.

All these topics have in common that they require sources that are strong synchrotron emitters. As it was found using ground VLBI observations, the most compact radio sources have a flat or inverted spectrum (the spectral flux increase with frequency), which is the result of self-absorption by relativistic electrons, or absorption by plasma surrounding the compact source. This, together with interstellar scattering, blurs the image of an object and sets the minimum resolution that can be obtained at a given wavelength. This effect is very strong towards the black hole at the center of our Galaxy, the source Sgr A*. However, the importance of these effects decreases significantly with frequency, and these effects are negligible in the sub-millimeter band. An additional advantage of the sub-millimeter band is that in that band there is an absolute minimum of the brightness temperature of the background radiation and therefore there one can obtain the best signal-tonoise ratio. At a wavelength of $250-500 \mu \mathrm{m}$ (frequency $600-1,200 \mathrm{GHz}$ ) there is a deep minimum of the spectral intensity $I_{v}$.

\section{Millimetron mission profile}

Millimetron is designed to provide high sensitivity, high to extremely high angular resolution and high spectral resolution as well as far-infrared imaging and spectroscopic capabilities. All these characteristics are essential in achieving the science goals outlined in the previous sections. High sensitivity and (extremely) high angular resolutions are achieved by using a $12 \mathrm{~m}$ diameter space antenna, either in single-dish mode or as element of a space-ground VLBI system. High spectral resolution is obtained by using heterodyne receivers, and the far-infrared imaging/ spectroscopy will be done by an imaging photometer/spectrometer. In order to achieve good u-v coverage in VLBI mode, an elliptical orbit is chosen.

\subsection{Launcher/orbit}

Launch services will be provided by Russia as the Millimetron mission is included in the Russian Federal Space Program. The Russian "Proton" type launcher in combination with a booster fully satisfies the requirements. The upgraded "Proton" heavy class launch vehicle is characterized by high power capacity. This type of launcher has been used for more than 30 years with very high reliability. The "Proton" payload fairing has been recently enlarged in order to double the space available for payloads. The Russian "Navigator" space platform will be used as basis of the spacecraft. Driven by the requirement of good u-v coverage in VLBI mode, an elliptical orbit with high apogee and an orbital period around the Earth of about 9.5 days is chosen. The perigee radius varies from 30.000 to $70.000 \mathrm{~km}$, and the apogee radius from 300.000 to $370.000 \mathrm{~km}$. Because of the orbit evolution, about $80 \%$ of 
the VLBI radio sources will be located on the sky close to the orbit plane projection at some time intervals, i.e. for such radio sources both very large and small baseline projections will provide the possibility to make good images with high and moderate angular resolution. The remaining $20 \%$ of the sources can be observed only with high angular resolution. The possibility of transit to a L2 orbit is being investigated.

\subsection{Scientific observing modes}

Millimetron has two basic observing modes:

(1) Single-dish submillimeter/far-infrared observations which do not differ fundamentally from observations with other space observatories like e.g. ISO or Herschel. The main difference is that space VLBI observations will take some of the available observing time, however, this amount is limited by the on-board data storage capacity and the required time for the down-link of the VLBI data. A demanding requirement is the pointing accuracy which for an antenna operating at submillimeter and far-infrared wavelengths needs to be better than 1 arc sec.

(2) Space-ground VLBI observations at (sub-) millimeter wavelengths allowing baselines more than 20 times the Earth diameter. SVLBI observing must be done simultaneously by the space radio telescope and ground-based radio telescopes. Due to the observing bandwidth $(2 \times 8 \mathrm{GHz})$, limitations in the onboard memory size (10 $\mathrm{Tb}$ foreseen) and the data transfer speed (1 Mbps), a VLBI observing session can last about $5 \mathrm{~min}$ before the data have to be downlinked for about $2.8 \mathrm{~h}$. Thus a total of $40 \mathrm{~min}$ VLBI observations per day are possible. During the data transfer single dish mode observations can be performed. It is envisaged that Millimetron will be working together with the following ground-based telescopes:

- Atacama Large Millimeter Array (ALMA). Millimetron will carry receivers for four bands fully compatible with ALMA. In first contacts between the Astro Space Center and ESO (the ALMA Executive in Europe), ESO has expressed interest in this possibility.

- Suffa radio telescope, a millimeter wave telescope of $70 \mathrm{~m}$ diameter which is being built on the Suffa plateau in Uzbekistan. It is planned that a dedicated part of telescope time will be used for SVLBI with Millimetron.

- Other telescopes including e.g. Pico Veleta (Spain), Plateau de Bure interferometer (France), HHT telescope in the US (Arizona), radio telescopes on Hawaii (USA) as well as the Green Bank and Effelsberg $100 \mathrm{~m}$ diameter telescopes.

\subsection{UV coverage in SVLBI mode}

The uv coverage of Millimetron is determined by the elliptical orbit with groundspace baselines of up to more than $300,000 \mathrm{~km}$. In order to improve the u-v coverage the technique of multi-frequency synthesis (MFS) can be used for continuum sources. Rapid frequency changes in the receiver will create more points in the $\mathrm{u}-\mathrm{v}$ plane for the same geometrical baseline. As an example, Fig. 3 shows the $u-v$ 
Fig. 3 u-v coverage of the Millimetron-ALMA interferometer for the source 1,334-127 using ALMA band six and multi-frequency synthesis

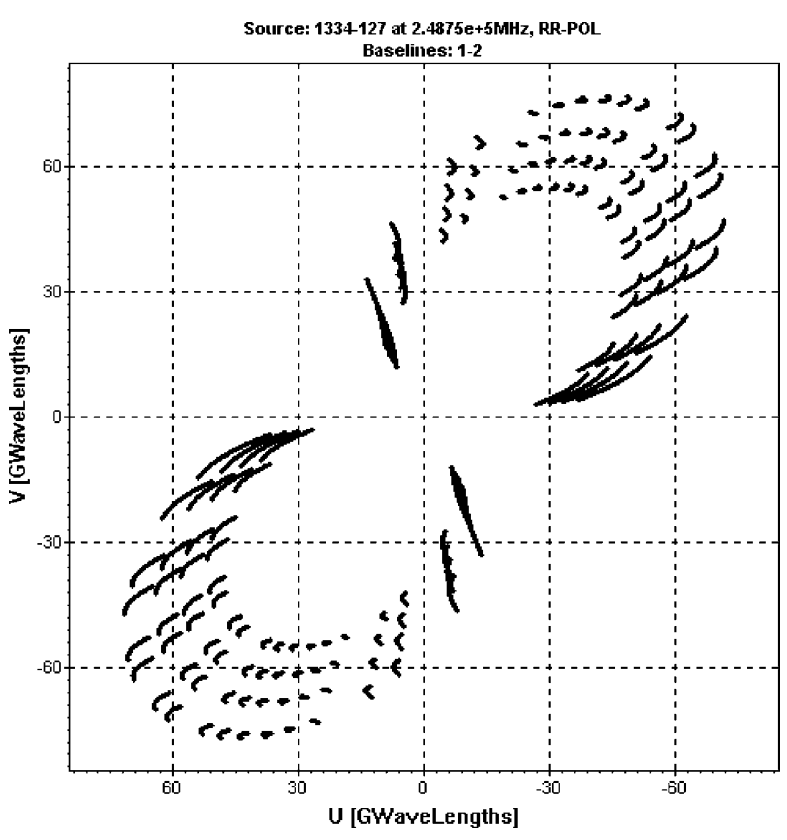

coverage for simultaneous observations between Millimetron and ALMA using frequency switching (MFS) within band six of ALMA (source 1334-127, 210-290 $\mathrm{GHz}$, four channels, 2 days of observations). The Millimetron orbit is perturbed by interactions with the Moon which allows to further improve the $\mathrm{u}-\mathrm{v}$ coverage if the same astronomical source is revisited after several months.

\subsection{Ground segment}

We distinguish between the Mission Ground Segment needed for mission operations and single-dish data transfer, and the VLBI Ground Segment for the VLBI data transfer. The Mission ground segment needs to provide daily contact with the satellite to exchange telemetry information, single dish observation data and the observing program and is similar to other scientific space missions. Due to the long orbital period global coverage will be required. The SVLBI ground segment will be responsible for SVLBI operations support. With three SVLBI tracking stations in the North (Pushchino near Moscow, provided by ASC), South (Tidbinbilla in Australia) and West (Green Bank, NRAO, USA) large part of the sky can be covered. The SVLBI tracking stations will need special VLBI equipment to fulfill their task.

\subsection{Critical issues}

A critical issue for a scientifically successful mission is achieving the $10 \mu \mathrm{m} \mathrm{rms}$ accuracy of the primary dish surface after the deployment of the $12 \mathrm{~m}$ antenna in space. The deployment itself will be demonstrated with the $10 \mathrm{~m}$ diameter RadioAstron antenna (launch planned for 2008/09) and is not seen as a high-risk item. However, in order to achieve the required surface accuracy for Millimetron, an 
active control system to adjust the (high-precision) petals is needed. Such a system requires development. We note that the James Webb Space Telescope (JWST) will use deployable, actively controlled mirror segments employing wavefront sensing techniques. For the Millimetron mirror operating in submillimeter wavelength bands, direct position measurement and control of the petals may be more attractive.

Another important item is the pointing accuracy which needs to be much better than 1 arc sec because of the small beams of Millimetron. This could be based on the Spitzer Space Telescope (better than 0.45 arc sec with a stability of $\sim 0.03$ arc sec over $600 \mathrm{~s}$ ), or an improved Herschel system.

For the space-ground VLBI mode, phase distortions of the signal traveling through the Earth atmosphere need to be corrected for. This can be done through monitoring the atmospheric emission around $183 \mathrm{GHz}$ (an atmospheric water line). The method is used very successfully at ground-based (sub-) millimeter interferometers and will be used at ALMA as well.

The behavior of the $12 \mathrm{~m}$ antenna under real operating conditions, i.e. at a physical temperature of $50 \mathrm{~K}$, will have to be investigated. Any approach should include at least three activities: (1) Accurate thermal and mechanical modeling of the whole telescope assembly, (2) testing of individual telescope petals (length $\sim 4.5 \mathrm{~m}$ ), and (3) testing of a scaled telescope model in an environmental chamber.

\section{Millimetron payload}

\subsection{Overview}

Table 2 gives an overview of all payload elements with the corresponding Technology Readiness Level (TRL) as used by space agencies to indicate the matureness of a certain technology.

Table 2 Payload elements for Millimetron

\begin{tabular}{|c|c|c|}
\hline Payload element & Main characteristics & TRL (as of Jun 07) \\
\hline Telescope & $\begin{array}{l}12 \mathrm{~m} \text { diameter, deployable, passively } \\
\text { cooled to } \sim 50 \mathrm{~K}, 10 \mu \mathrm{m} \text { overall } \\
\text { accuracy, } 2 \mu \mathrm{m} \text { central } 3.5 \mathrm{~m}\end{array}$ & $\begin{array}{l}\text { TRL }=6 \text { for deployment } \\
\text { mechanism based on } \\
\text { RadioAstron mission }\end{array}$ \\
\hline Heat shields & Multi-layer membrane, deployable & $\mathrm{TRL}=4$ for JWST sunshield \\
\hline $\begin{array}{l}\text { VLBI Instrumentation: } \\
\text { 18-26 GHz receiver }\end{array}$ & Heterodyne front-end & RadioAstron receiver has $\mathrm{TRL}=6$ \\
\hline $\begin{array}{l}\text { VLBI instrumentation: } \\
\text { ALMA bands }\end{array}$ & $\begin{array}{l}\text { Receivers at ALMA bands } 1,3,6 \text {, } \\
\text { and 9; with ALMA compatible } \\
\text { characteristics }\end{array}$ & $\begin{array}{l}\text { Based on ALMA development } \\
\text { with TRL }=6\end{array}$ \\
\hline $\begin{array}{l}\text { Single-dish instrumentation: } \\
\text { submm heterodyne receivers }\end{array}$ & $\begin{array}{l}\text { Dual polarization receivers in bands } \\
\text { around } 557 \mathrm{GHz}, 1,900 \mathrm{GHz} \text {, and } \\
4,700 \mathrm{GHz}\end{array}$ & $\begin{array}{c}557 \text { and } 1,900 \mathrm{GHz} \text { based on } \\
\text { Herschel-HIFI with TRL }=6\end{array}$ \\
\hline $\begin{array}{l}\text { Single-dish instrumentation: } \\
\text { far-IR spectrometer }\end{array}$ & $\begin{array}{l}\text { Imaging photometer/spectrometer } \\
\text { covering } \lambda 60-210 \mu \mathrm{m}\end{array}$ & $\begin{array}{l}\text { Based on Herschel-PACS with } \\
\text { TRL }=6\end{array}$ \\
\hline Closed-cycle coolers to $4 \mathrm{~K}$ & $\begin{array}{l}\text { Capable to cool instrument suite, } 5 \\
\text { year lifetime }\end{array}$ & $\begin{array}{l}\mathrm{TRL}=6 \text { for } 20 \mathrm{~mW} \text { cooling } \\
\text { (Planck) }\end{array}$ \\
\hline VLBI mass memory & $\geq 10 \mathrm{~Tb}$ & $\mathrm{TRL}=8$ for $3 \mathrm{~Tb}$ \\
\hline
\end{tabular}


Table 3 Millimetron instrument interface and resource summary

\begin{tabular}{|c|c|c|}
\hline Parameter & Baseline requirement/specification & Comments \\
\hline Instrument suite mass & $\begin{array}{l}<500 \mathrm{~kg} \text { total, distributed over focal } \\
\text { plane and service module }\end{array}$ & $\begin{array}{l}\text { Overall mass of single-dish and VLBI } \\
\text { instrumentation including warm } \\
\text { electronics }\end{array}$ \\
\hline Instrument suite power & $<1400 \mathrm{~W}$ & $\begin{array}{l}\text { Total available power for instrument } \\
\text { suite on the Russian "Navigator" } \\
\text { spacecraft incl. cooler }\end{array}$ \\
\hline Instrument suite volume & $2.7 \mathrm{~m}^{3}$ & \\
\hline Instrument suite cooling & $\begin{array}{l}50 \mathrm{~mW} \text { at } 4 \mathrm{~K}, 200 \mathrm{~mW} \text { at } 20 \mathrm{~K}, 2 \mathrm{~W} \\
\text { at } 70 \ldots 100 \mathrm{~K}\end{array}$ & $\begin{array}{l}\text { Closed-cycle cryo-cooler to provide } \\
\text { instrument cooling, first rough estimate }\end{array}$ \\
\hline Telemetry & $80 \mathrm{~Tb}$ daily $\mathrm{TM}$ & Requires $1 \mathrm{Mbps}$ link \\
\hline Pointing & $<1$ " required & "Navigator" spacecraft to be modified \\
\hline
\end{tabular}

Based on the experience with instruments for Herschel, RadioAstron and ALMA, an estimate of the instrument suite requirements (single-dish and SVLBI) on spacecraft resources in given in Table 3. These estimates will have to be refined as part of further studies. In particular, the available power for active cooling to $4 \mathrm{~K}$ will most likely be the primary limitation for the number of receiving channels of the instrument suite (with mass, volume and electrical power being secondary limitations).

\subsection{The $12 \mathrm{~m}$ space telescope}

Optical and mechanical design parameters The optical design of the telescope (Fig. 4) is a classical two-mirror Cassegrain type. The need for a low physical temperature $(\leq 50 \mathrm{~K})$ telescope requires minimization of the exterior thermal radiation loading driving the telescope design to a two-mirror system with the secondary mirror "shielded" by the deep main mirror. The reflective surface of the main mirror is formed by a central solid mirror (3.5 m diameter with high surface accuracy) and outer petals unfolded after launch. It will be made out of $\mathrm{C}-\mathrm{C}$ or $\mathrm{Si}-\mathrm{C}$ (possibly in combination) with metallic (Gold) reflective coating. The main parameters of the deployable space telescope are: main dish diameter $12,000 \mathrm{~mm}$, main dish focal length

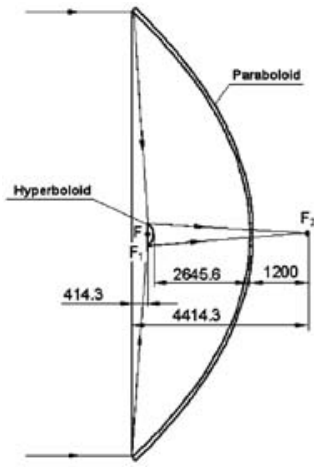

a)

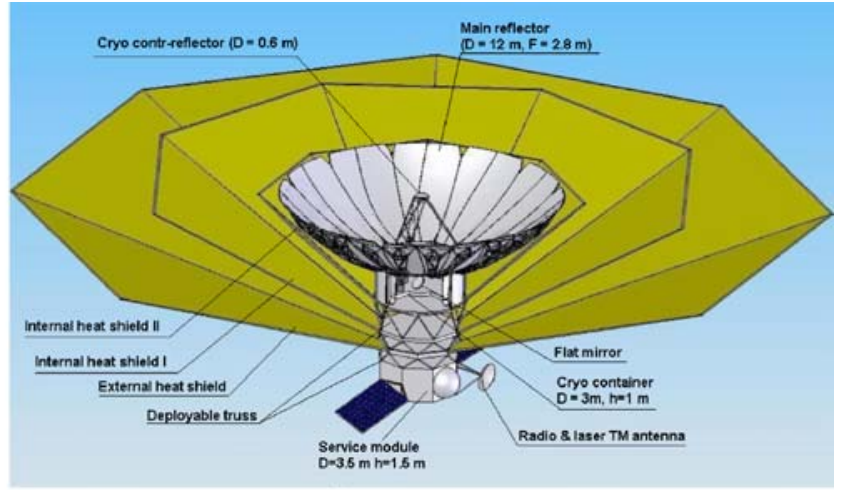

b)

Fig. 4 Millimetron $12 \mathrm{~m}$ telescope. a Main distances. b Layout 
2,800 mm, main dish surface accuracy $10 \mu \mathrm{m}(3 \mu \mathrm{m}$ for the central $3.5 \mathrm{~m}$ solid part of the main dish), secondary dish diameter $600 \mathrm{~mm}$, total focal length of the system $81,550.7 \mathrm{~mm}$.

Special measures have to be taken to achieve the required telescope surface accuracy. An active surface control system will be employed including either actuators in the petals' support structure or active surface elements in the main optical path and main surface shape measurement system. The active control system will mainly compensate for inaccuracies in the deployed petals positions and variations of the overall surface due to temperature changes. Actuators in the secondary mirror support structure will allow refocusing of the telescope by mirror displacement. Passive radiation cooling will be used to achieve a telescope physical temperature of $\sim 50 \mathrm{~K}$.

Deployment strategy The main mirror design and deployment strategy is based on the "RadioAstron" project experience. "RadioAstron" has a $10 \mathrm{~m}$ diameter deployable mirror (Fig. 5) which as of 2007 is under vibration flight qualification tests and is being readied for launch in 2008. Deployment of the telescope is made by one drive and is based on a rocker-lever mechanism. Different stages of the telescope deployment are shown in Fig. 6. The launch packaging is shown at the left and the fully open telescope is shown at the right. The petals will be mechanically joined at the outer edge of the telescope providing higher accuracy of deployment. The length of the Millimetron petals is $4.3 \mathrm{~m}$ and determines the height of the telescope package in the fairing. An active surface control system will be responsible for the correction of residual errors of the panel positions.

Heat shielding concept The telescope surface will be passively cooled to a temperature of $\sim 50 \mathrm{~K}$. The basic thermal concept of the Millimetron telescope is shown in Fig. 7. In order to protect the surfaces of the primary and secondary mirrors from the thermal loading by the Sun, Earth, Moon and spacecraft itself, deployable multi layer heat screens in the form of cones can be used. The first screen diameter is $30-35 \mathrm{~m}$, the second $20-25 \mathrm{~m}$, the third (auxiliary) $13-15 \mathrm{~m}$. The screens could be made of metallized film similar to Kapton. The first screen has two layers. All film layers have double-sided metallization. Preliminary thermal modeling indicates the following average temperatures of the screens: First screen one layer-less then $350 \mathrm{~K}$, two layer-less than $250 \mathrm{~K}$; Second screen-less then $150 \mathrm{~K}$; Third screen-less than $50 \mathrm{~K}$.

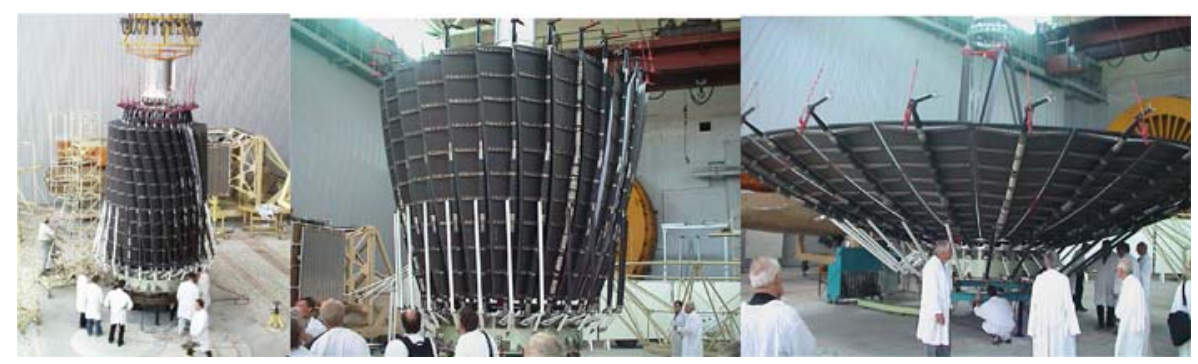

Fig. 5 Different stages of the "RadioAstron" $10 \mathrm{~m}$ antenna deployment 


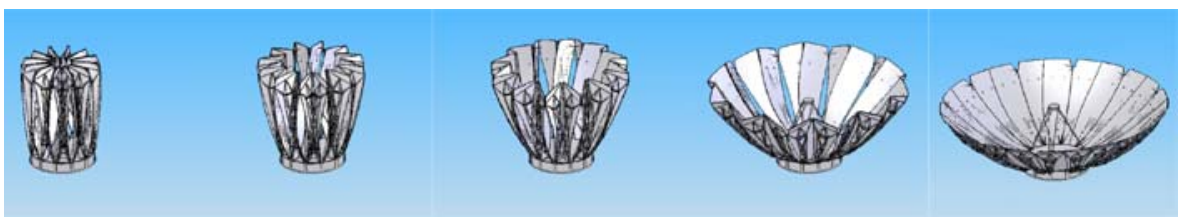

Fig. 6 Stages of the Millimetron telescope deployment. Left-launch packaging, right-operational telescope

\section{Scientific instrumentation}

Here we describe a baseline instrumentation plan for the two basic scientific modes of operation (single-dish and SVLBI). The final instrument suite will depend to a large degree on the available spacecraft resources with $4 \mathrm{~K}$ cooling power probably being the most critical parameter. An assessment study needs to refine the instrument technical requirements and evaluate various trade-off options.

The instrumentation would be built by a European consortium of scientific and technical institutes similar to the Herschel and ALMA schemes. All the required expertise and experience is readily available, and many institutions of the present Herschel and ALMA consortia have already expressed interest to participate.

\subsection{Single-dish instrumentation}

The baseline instrumentation for the single-dish observing mode consists of the two heterodyne instruments HET-1 and HET-2 and the Millimetron Photodetector Array Camera and Spectrometer (M-PACS). HET-1, a dual-frequency superconductorinsulator-superconductor (SIS) array, will operate around the astrophysically important frequencies 557 and 1,100 GHz, and HET-2, a three-channel hot electron bolometer (HEB) receiver, will cover 1.9, 2.7 and $4.7 \mathrm{THz}$ (the latter using the central $3.5 \mathrm{~m}$ high-precision part of the antenna). M-PACS will be an adapted copy of Herschel-PACS with improved detectors covering the wavelength regime 60 to $210 \mu \mathrm{m}$. The instrument and detector technologies as well as operational modes and calibration schemes can build heavily on the developments done for Herschel. The technical risk is accordingly low.

Table 4 lists the key characteristics of the Millimetron science instrumentation for single-dish observations.

HET-1 and HET-2 are heterodyne receivers providing very high spectral resolution $\left(\geq 10^{6}\right)$ in combination with a Fast-Fourier-Transform spectrometer (FFTS). The channels up to 2,000 $\mathrm{GHz}$ can directly build on the successful developments for the Herschel-Heterodyne Instrument for the Far-infrared (HIFI), whereas the higher

Fig. 7 Preliminary scheme of thermal screens for Millimetron

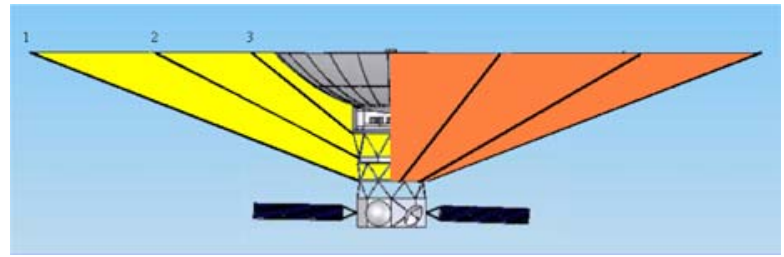


Table 4 Millimetron single-dish instrumentation key characteristics

\begin{tabular}{|c|c|c|c|c|c|c|}
\hline Instrument & $\begin{array}{l}\text { Frequency }(\mathrm{GHz}) \\
\text { or wavelength }\end{array}$ & $\begin{array}{l}\text { Angular } \\
\text { resolution (") }\end{array}$ & $\begin{array}{l}\text { Spectral } \\
\text { resolution }\end{array}$ & $\begin{array}{l}\text { Detector } \\
\text { technology }\end{array}$ & Sensitivity & TRL \\
\hline \multicolumn{7}{|c|}{ Heterodyne receivers } \\
\hline HET-1 & $\begin{array}{c}480-700 \\
1,100-1,400\end{array}$ & $\begin{array}{l}8 \ldots 12 \\
5 \ldots 6\end{array}$ & $\begin{array}{l}\geq 10^{6} \\
\geq 10^{6}\end{array}$ & $\begin{array}{l}\text { SIS } 2 \times 2 \text { mixer array } \\
\text { with multiplier LO }\end{array}$ & $\begin{array}{l}T_{\text {sys }}<100 \mathrm{~K} \\
T_{\text {sys }}<200 \mathrm{~K}\end{array}$ & $\begin{array}{l}6 \\
6\end{array}$ \\
\hline HET-2 & $\begin{array}{l}1,650-2,000 \\
2,600-2,700 \\
4,700-4,800\end{array}$ & $\begin{array}{l}\sim 3 \\
\sim 2.5 \\
4^{\mathrm{a}}\end{array}$ & $\begin{array}{l}\geq 10^{6} \\
\geq 10^{6} \\
\geq 10^{6}\end{array}$ & $\begin{array}{l}\text { HEB mixers with } \\
\text { multiplier or QCL LO }\end{array}$ & $\begin{array}{l}T_{\text {sys }}<500 \mathrm{~K} \\
T_{\text {sys }}<700 \mathrm{~K} \\
T_{\text {sys }}<1,000 \mathrm{~K}\end{array}$ & $\begin{array}{l}6 \\
4 \\
4\end{array}$ \\
\hline \multicolumn{7}{|c|}{ Far-Infrared imaging photometer/spectrometer } \\
\hline M-PACS & $60-210 \mu \mathrm{m}$ & $\geq 4$ & $\begin{array}{l}\text { Few } 10^{3} \\
\text { spectrom. }\end{array}$ & $\begin{array}{l}\text { Photoconductor } \\
\text { arrays }\end{array}$ & $2 \times 10^{-18} \mathrm{Wm}^{-2}$ & 6 \\
\hline
\end{tabular}

${ }^{a}$ Using the central $3.5 \mathrm{~m}$ dish

frequency channels of HET-2 around 2.7 and 4.7 THz, unique to Millimetron, have been demonstrated in the lab. For HET-1 it is planned to use $2 \times 2$ SIS waveguide mixer arrays with multiplier chain local oscillators (LO). This type of technology has been developed for many ground-based telescopes and as single mixers for Herschel-HIFI. The $2 \times 2$ mixer arrays for Millimetron would be a new development for space which could heavily build on ground-based developments. It is interesting to note that multiplier LOs up to $2,000 \mathrm{GHz}$ are now becoming available commercially and at much lower cost than for HIFI. HET-1 will make use of demonstrated SIS technology using $\mathrm{Nb}-\mathrm{AlN}-\mathrm{Nb}$ tunnel junctions in combination with $\mathrm{Nb}-\mathrm{SiO}_{2}-\mathrm{Nb}$ on-chip tuning elements as used at all major submillimeter observatories and in Herschel-HIFI.

HET-2 will in principle employ hot electron bolometer mixers (HEBM) as used in HIFI. However, due to sensitivity and intermediate frequency (IF) bandwidth limitations of HEBM, it is attractive to develop SIS junction technology up to 2,000 $\mathrm{GHz}$ by utilizing high energy gap superconducting materials. If successful, this SIS technology will allow to achieve better sensitivity and IF frequency coverage. The best technology for the 1,650-2,000 GHz channel of HET-2 can be selected depending on development result. The 2.7 and $4.7 \mathrm{THz}$ channels will in any case use HEB mixers. Local oscillators for HET-2 will be multiplier chains and quantumcascade-lasers (QCL). QCLs have been used in the lab as THz LO source. Some development is required for the QCL to stabilize its frequency and reduce the dissipated power at the $30 \ldots 70 \mathrm{~K}$ level.

Several developments of HEB mixers and mixer arrays are underway for frequencies beyond $2 \mathrm{THz}$. A heterodyne receiver at $2.8 \mathrm{THz}$ using a quasi-optical HEB mixer and QCL local oscillator has been demonstrated with a noise temperature of 1,400 K (DSB) [1]. Another interesting development is work carried out under ESA contract nr. 16940 at Chalmers University (Sweden), LERMA (Observatoire de Paris) and LAAS (Toulouse) to build a $4 \times 4$ pixel array at $2.5 \mathrm{THz}$ (called SHAHIRA) using membrane-based HEB mixers. Figure 8 shows a first prototype of this 16-pixel array ready for device integration.

Heterodyne back-end The heterodyne receivers will share a FFTS backend which is a digital spectrometer providing wide instantaneous bandwidth with high spectral 
Fig. $84 \times 4$ pixel $2.5 \mathrm{THz} \mathrm{HEB}$ mixer array prototype

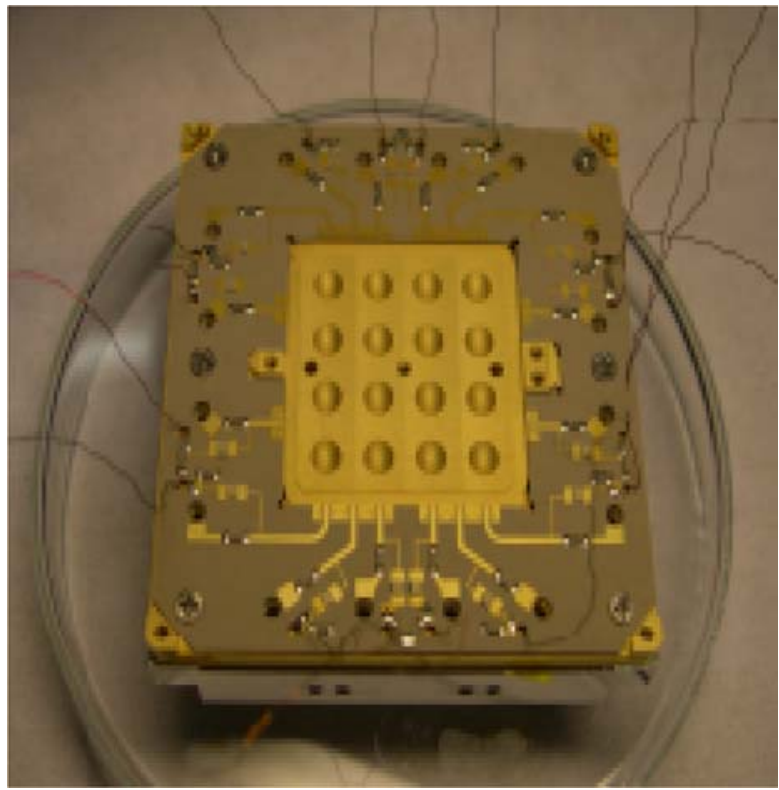

resolution. In the case of a $2 \times 2$ pixel receiver (dual polarization) with an IF bandwidth of $4 \mathrm{GHz}$ each, a total spectrometer bandwidth of $32 \mathrm{GHz}$ is required. Current FFT technology $(\mathrm{TRL}=6)$, field-proven in now almost 2 years of continuous operation at, e.g., the APEX submillimeter telescope, provides an instantaneous bandwidth of $1 \mathrm{GHz}$ with 8-16 k channels. Under development at MPIfR (Germany) are single-board FFTs with $1.5 \mathrm{GHz}$ bandwidth/16 k (readiness mid 2007) and, using the most recent ADC available, $2.5 \mathrm{GHz} / 8 \mathrm{k}$ channels (spring 2008). With the latter implementation of a hybrid backend with $3 \times 2.5 \mathrm{GHz}$ to combine to a total of $\sim 7$ $\mathrm{GHz}$ (allowing for some overlap) bandwidth is within reach. The enormous increase of ADC bandwidth during the last years makes it very likely that FFTS can be pushed to instantaneous bandwidths wider than $3 \mathrm{GHz}$ in the near future, thereby further reducing the complexity of the backend. The spectral resolution (the number of channels) that can be achieved is basically constrained by the on-board resources (power dissipation) and the level of complexity that appears acceptable for a space mission. Field-programmable gate arrays have quite a long space heritage. In any case, digital FFT spectrometers can provide the back-end capacities required by the Millimetron mission concept. Some development work will be needed to increase bandwidth, to optimize operation to minimum power dissipation, to adapt to particular constraints of a space observatory, and to comply with space qualification requirements.

The Far-infrared photometer and spectrometer M-PACS can be based on the successful development of the PACS instrument, as built for Herschel which offers photometric and spectroscopic capabilities in the wavelength band from 60-210 $\mu \mathrm{m}$ :

A) Imaging dual-band photometry (60-85 or 85-130 and 130-210 $\mu \mathrm{m})$ over a field of view of $1.75^{\prime} \times 3.5^{\prime}$, with full sampling of the telescope point spread function. 
B) Integral-field line spectroscopy between 57 and $210 \mu \mathrm{m}$ with a resolution of $175 \mathrm{~km} / \mathrm{s}$ and an instantaneous coverage of $1,500 \mathrm{~km} / \mathrm{s}$, over a field of view of $47^{\prime \prime} \times 47^{\prime \prime}$.

Both modes will allow spatially chopped observations by means of an instrumentinternal chopper mirror with variable throw.

The focal plane unit provides these capabilities through five functional units:

1. Common input optics with the chopper, calibration sources and a focal plane splitter.

2. The photometer optical train with a di-chroic beam splitter and separate reimaging optics for the two short wavelength bands $(60-85 / 85-130 \mu \mathrm{m})$ and the long-wavelength band (130-210 $\mu \mathrm{m})$, respectively; band-defining filters on a wheel select one of the two short-wavelength bands at a time.

3. The spectrometer optical train with an image slicer unit for integral field spectroscopy, an anamorphic collimator, a diffraction grating in Littrow mount with associated actuator and position readout, anamorphic re-imaging optics, and a di-chroic beam splitter for separation of diffraction orders.

4. Two filled silicon bolometer arrays with $16 \times 32$ and $32 \times 64$ pixels, with cryogenic buffers/multiplexers and a common $0.3 \mathrm{~K}$ sorption cooler, for simultaneously imaging in two bands, $60-85$ or $85-130$ and $130-210 \mu \mathrm{m}$ over a field of view of $1.75^{\prime} \times 3.5^{\prime}$

5. Two Ge:Ga photoconductor arrays (stressed and unstressed) with $16 \times 25$ pixels each, that allow to perform imaging line spectroscopy over a field of $50 " \times 50$ ", resolved into $5 \times 5$ pixels, with an instantaneous spectral coverage of $1,500 \mathrm{~km} / \mathrm{s}$ and a spectral resolution of $175 \mathrm{~km} / \mathrm{s}$, with sensitivities $(5 \sigma$ in $1 \mathrm{~h})$ of $4 \mathrm{mJy}$ or $3-20 \times 10^{-18} \mathrm{~W} / \mathrm{m}^{2}$.

Both the heterodyne instruments HET-1/HET-2 and M-PACS need cooling to $\sim 4$ $\mathrm{K}$ from the spacecraft instrument cooling system (analogue to Herschel) with $\mathrm{M}$ PACS having its own internal cooler to $0.3 \mathrm{~K}$.

\subsection{Space segment VLBI instrumentation}

Overview Figure 9 describes in general terms what is needed for performing SVLBI. The signal from the telescope is received by one of the SVLBI front ends and down converted to an intermediate frequency which is filtered and conditioned in the IF processor unit and then digitized. Currently quantization of two bits over a $4-8 \mathrm{GHz}$

Fig. 9 Layout of SVLBI instrumentation package

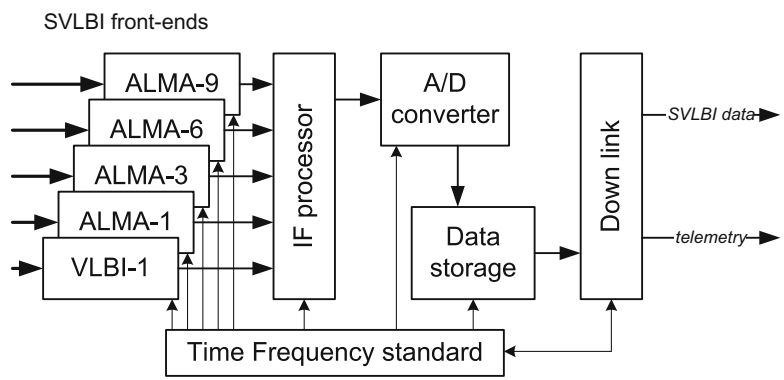


band with dual polarization is considered which would result in a data rate of $16 \mathrm{~Gb} / \mathrm{s}$. The digital signals are recorded in a data storage unit which should have sufficient capacity to hold approx $40 \mathrm{~min}$ of SVLBI data (10 TB capacity). A high-speed downlink transfers the data to the ground.

SVLBI front-ends The front end instrumentation for the VLBI observing mode will consist of a low frequency $(18-26 \mathrm{GHz})$ front-end very similar to the one which will be flown on the Russian RadioAstron mission in 2008/09. This receiver on Millimetron will greatly improve the $\mathrm{u}-\mathrm{v}$ plane coverage achieved by RadioAstron and is required to ensure proper cross calibration. Unique to Millimetron will be the VLBI receivers at the ALMA frequency bands one $(31.3-45 \mathrm{GHz})$, three (84-116 $\mathrm{GHz})$, six (211-275 GHz) and nine (600-720 GHz). Table 5 summarizes the key characteristics of the Millimetron VLBI instrumentation.

The VLBI-1 receiver will be based on previous developments as carried out for many radio telescopes and the RadioAstron or VLBI Space Observatory Program missions. The ALMA bands will follow technology developed within the ALMA project for sideband separating SIS mixers, IF amplifiers and LOs. Adaptation of the ALMA technology for space use should not pose much difficulty as it is conceptually similar to the one used for Herschel (in fact ALMA uses some components that were developed for HIFI). Special attention will be paid to the polarization response of these instruments as observing polarization is one of the scientific goals. All ALMA band mixers require cooling to $4 \mathrm{~K}$ like the HET-1 and HET-2 instruments.

On board time and frequency standard There are two possibilities for onboard time synchronization. The first one is a hydrogen frequency standard developed in Russia which will be flown on the RadioAstron mission. The second one is an optical frequency standard with a stability of $3 \times 10^{-16}$ in $5 \mathrm{~min}$. This optical standard is under construction at the Lebedev Physical Institute. The package will be delivered by the Russian Space Agency.

$A / D$ converter and data storage The $\mathrm{A} / \mathrm{D}$ converter technology will be the same as used for the FFTS backends. Adequate IF bandwidth can be covered by current technology already (ALMA). More weight/energy efficient solutions are expected to appear in the near future. On board data storage modules of up to several TBit are

Table 5 VLBI instrumentation key characteristics

\begin{tabular}{|c|c|c|c|c|c|}
\hline \multirow{2}{*}{$\begin{array}{l}\text { Instrument type } \\
\text { Receiver }\end{array}$} & \multirow{2}{*}{$\begin{array}{l}\text { VLBI } \\
\text { receiver } \\
\text { VLBI-1 }\end{array}$} & \multicolumn{4}{|c|}{ Heterodyne receivers covering the ALMA bands } \\
\hline & & ALMA-1 & ALMA-3 & ALMA-6 & ALMA-9 \\
\hline Frequency range & $18-26 \mathrm{GHz}$ & $31.3-45 \mathrm{GHz}$ & $84-116 \mathrm{GHz}$ & $211-275 \mathrm{GHz}$ & $600-720 \mathrm{GHz}$ \\
\hline Detector technology & $\begin{array}{l}\text { HEMT } \\
\text { amplifier }\end{array}$ & $\begin{array}{l}\text { SIS mixers, } \\
\text { multiplier chain } \\
\text { LO, dual } \\
\text { polarization }\end{array}$ & $\begin{array}{l}\text { SIS mixers, } \\
\text { multiplier chain } \\
\text { LO, dual } \\
\text { polarization }\end{array}$ & $\begin{array}{l}\text { SIS mixers, } \\
\text { multiplier chain } \\
\text { LO, dual } \\
\text { polarization }\end{array}$ & $\begin{array}{l}\text { SIS mixers, } \\
\text { multiplier chain } \\
\text { LO, dual } \\
\text { polarization }\end{array}$ \\
\hline Sensitivity $T_{\text {sys }}$ & $<40 \mathrm{~K}$ & $<17 \mathrm{~K} \mathrm{SSB}$ & $<37 \mathrm{~K} \mathrm{SSB}$ & $<90 \mathrm{~K} \mathrm{SSB}$ & $<150 \mathrm{~K}$ DSB \\
\hline TRL & 6 & 6 & 6 & 6 & 6 \\
\hline
\end{tabular}


available commercially and space qualified from e.g. European Aeronautic Defense and Space Company (EADS)-Astrium. It is anticipated that the maximum available capacity will only grow over the years. $10 \mathrm{~TB}$ would be sufficient for the mission but expanding on the capacity up to $30 \ldots 40$ TB will benefit the mission.

Down link The high speed down link is a crucial part of the SVLBI system. Its speed will define the ground tracking station load for data transfer. The currently demonstrated radiolink data rate is two channels of $512 \mathrm{Mb} / \mathrm{s}$. It will take $22 \mathrm{~h}$ to transmit $10 \mathrm{~TB}$ to Earth. A development towards improving data rates by using optical and other means would be of a great benefit for the Millimetron mission.

Telemetry and time synchronization data links can be common for the single dish and the SVLBI mode as they do not have high requirements concerning the data rate. Current state-of-the-art satellite communication equipment cold be used (similar to Herschel).

\subsection{Special requirements}

Instrument cooling to $4 \mathrm{~K}$ The planned minimum mission life time of 5 years calls for a closed-cycle cryo-cooler to $4 \mathrm{~K}$, also providing cooling to $20 \mathrm{~K}$ and $70 \ldots 100 \mathrm{~K}$ levels. The temperatures below $1 \mathrm{~K}$ required for the M-PACS detectors will be generated within the M-PACS instrument (similar to the Herschel PACS scheme). A preliminary estimate of the required cooling powers for the scientific instrumentation gives: $40-50 \mathrm{~mW}$ at $4 \mathrm{~K}, 200 \mathrm{~mW}$ at $20 \mathrm{~K}$, and $2 \mathrm{~W}$ at $70 \ldots 100 \mathrm{~K}$, with a temperature stability of $5 \mathrm{mK}$ at $4 \mathrm{~K}, 10 \mathrm{mK}$ at $20 \mathrm{~K}$, and $0.2 \mathrm{~K}$ at $70 \ldots 10 \mathrm{~K}$. These values are first estimates based on the specifications and experience with Herschel and ALMA instruments and will have to be refined during a study phase.

Mass storage memory The SVLBI observing mode generates large amounts of data which will have to be stored on-board before down-link. The duration of SVLBI observations depends on the available mass storage memory and the IF bandwidth of the recorded data. For an IF bandwidth of $2 \times 8 \mathrm{GHz}$ (dual polarization) of an ALMA band and $300 \mathrm{~s}$ of observations, about $10 \mathrm{~Tb}$ of memory are needed. ESA's Sentinel2 mission will fly $2 \mathrm{~Tb}$ of memory (provided by EADS-Astrium), and presently modules of $3 \mathrm{~Tb}$ are commercially available. It is anticipated that the rapid development of memory technology and density will continue, and that within the coming years (mostly driven by commercial applications) larger amounts of memory for space applications will become available.

\section{Basic spacecraft key factors}

The spacecraft platform for the Millimetron mission will be supplied by the Russian Space Agency. It will largely be based on the general purpose space platform "Navigator" which is built by the Lavochkin Association. The basic layout of the "Navigator" spacecraft is shown in Fig. 10. It will provide attitude control, power and control/telemetry communications to the ground segment. The scientific payload 

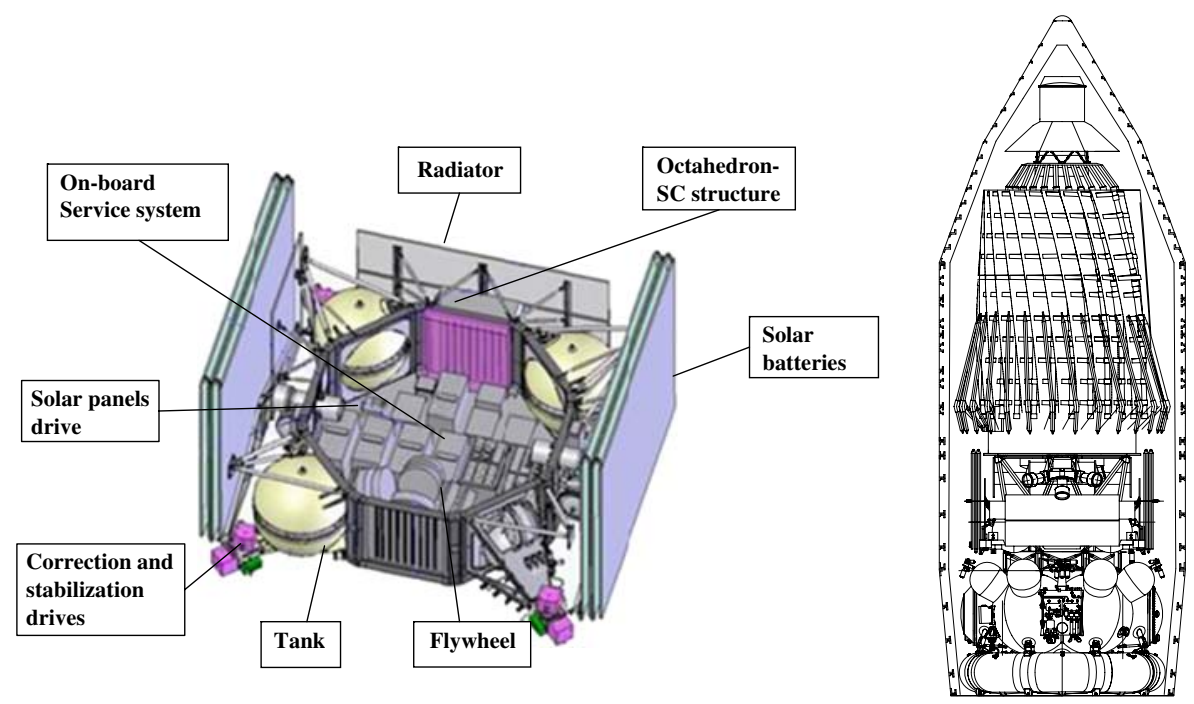

Fig. 10 Layout of the space platform "Navigator" (left) and the instrument complex of "RadioAstron" mounted on the "Navigator" platform in the Proton launcher fairing (right)

instruments, telescope and high speed communication link will be mounted on a mechanical interface provided by the platform. The "Navigator" platform will be used for the upcoming space missions "Electro" (launch in 2008), "RadioAstron" (launch in 2008) and other scientific missions. Some of the underlying systems of this platform have already been flown successfully. A few parameters of the "Navigator" platform will require modification for Millimetron: the available power, payload mass and pointing/stabilization accuracy need to be increased.

\section{Key technology areas}

The key technology areas of Millimetron are listed in Table 6. The most critical item appears to be the control of the $12 \mathrm{~m}$ dish surface after launch and deployment to

Table 6 Key technology areas for Millimetron

\begin{tabular}{llll}
\hline Payload element & Technology area & TRL & Comment \\
\cline { 2 - 3 } Telescope & Telescope petals & $3-4$ & Russian Space Agency \\
& Deployment strategy & 6 & Based on Russian RadioAstron telescope \\
& Active surface control & $2-4$ & Development by Astro Space Center \\
Spacecraft & Heat shields & $4-6$ & Developed for JWST \\
Scientific & Heterodyne and bolometer & $5-6$ & By "Lavochkin Association" \\
instrumentation & instrument for single-dish operation & & Spin-off from Herschel \\
& Heterodyne receivers for VLBI & 6 & Spin-off from ALMA \\
& Cryo-cooler & $4-6$ & Cryo-cooler programs at ESA, NASA, \\
& & & and JAXA; TRL 6 is for Planck \\
& & & compatible cooling power \\
& VLBI mass storage memory & $6-7$ & Available from EADS-Astrium \\
\hline
\end{tabular}


achieve the $10 \mu \mathrm{m}$ surface accuracy. All other key items are demonstrated at different levels (lab, ground, space, or flight proven).

\section{References}

1. Gao, J.R., et al.: Appl. Phys. Lett 86, 244104(2005)

2. Glover, S.C.O., and Mac Low, M.M.: APJ 659, 1317(2007)

3. Helmich, F.P., and Ivison, R.: this issue

4. Hennebelle, P., Audit, E., Miville-Deschênes, M.-A.: A\&A 465, 445 (2007)

5. Hily-Blant, P., Falgarone, E.: A\&A 469, 173 (2007)

6. Mizusawa, H., Omukai, K., Nishi, R.: PASJ 57, 951 (2005)

7. Neufeld, D.A., Green, J.D., Hollenbach, D.J., et al.: APJ 647, 33 (2006)

8. Papadopoulos, P.P., Isaak, K.G., van der Werf, P.P.: APJ 668, 815 (2007) 